doi:10.13108/2021-13-3-174

\title{
PERTURBATION THEORY METHODS IN PROBLEM OF PARAMETRIC RESONANCE FOR LINEAR PERIODIC HAMILTONIAN SYSTEMS
}

\author{
M.G. YUMAGULOV, L.S. IBRAGIMOVA, A.S. BELOVA
}

\begin{abstract}
We consider the problem on parametric resonance for linear periodic Hamiltonian systems depending on a small parameter. We propose new formulae based on the methods of the perturbation theory for linear operators in the problem on approximate construction of multipliers for linear non-autonomous periodic Hamiltonian systems. We focus on obtaining the formulae for the first correctors of perturbations of multiple definite and indefinite multipliers. The proposed formulae lead to new Lyapunov stability criteria for linear periodic Hamiltonian systems in critical cases. We consider applications to the problem on parametric resonance in main resonances. The obtained results are formulated in terms of the original equations and lead us to effective formulae and algorithms. The effectiveness of the proposed formulae is demonstrated by solving the problem of plotting the boundaries of the stability regions of triangular libration points of a planar bounded elliptic three-body problem.
\end{abstract}

Keywords: Hamiltonian system, stability, multiplier, small parameter, parametric resonance, perturbation theory, three-body problem, libration point.

Mathematics Subject Classification: 37J25, 37J40, 37N05

\section{INTRODUCTION}

1.1. Main equations. We consider a linear periodic Hamiltonian system (LPHS) depending on small scalar or vector parameter $\varepsilon$ :

$$
\frac{d x}{d t}=J A(t, \varepsilon) x, \quad x \in \mathbb{R}^{2 N},
$$

in which $A(t, \varepsilon)$ is a real symmetric $T$-periodic in $t$ matrix, that is, $A(t+T, \varepsilon) \equiv A(t, \varepsilon)$, while the matrix $J$ is defined by the identity:

$$
J=\left[\begin{array}{cc}
0 & I \\
-I & 0
\end{array}\right]
$$

here $I$ is the unit $N \times N$ matrix. We assume that

- the entries of the matrix $A(t, \varepsilon)$ are continuous in $t$ and $\mathbb{C}^{k}$-smooth in $\varepsilon$, where $k \geqslant 1$;

- the identity holds:

$$
A(t, 0) \equiv A_{0},
$$

where $A_{0}$ is a constant symmetric matrix.

M.G. Yumagulov, L.S. Ibragimova, A.S. Belova, Perturbation theory methods in problem of PARAMETRIC RESONANCE FOR LINEAR PERIODIC HAMILTONIAN SYSTEMS.

(C) Yumagulov M.G., Ibragimova L.S., Belova A.S. 2021.

The research of the third author is made in the framework of State Task of the Ministry of Science and Higher Education of Russian Federation (code of scientific theme FZWU-2020-0027).

Submitted February 18, 2021. 
Condition (1.3) that as $\varepsilon=0$, LPHS (1.1) is a linear autonomous Hamiltonian system (LAHS):

$$
\frac{d x}{d t}=J A_{0} x, \quad x \in \mathbb{R}^{2 N} .
$$

We call system (1.4) unperturbed, while (1.1) is called perturbed system.

In the paper we discuss some issues on constructing the formulae for the first corrector while perturbing multiple definite and indefinite multipliers of system (1.4). We consider their applications to studying the problem on a parametric resonance in system (1.1). A more detailed formulation will be given in what follows.

1.2. Auxiliary facts. We first briefly some auxiliary facts from the general theory of linear Hamiltonian systems, see, for instance, [1]-[4].

We consider a linear periodic Hamiltonian system:

$$
\frac{d x}{d t}=J A(t) x, \quad x \in \mathbb{R}^{2 N}
$$

here $A(t)$ is a real symmetric matrix, the entries of which are continuous and $T$-periodic in $t$ functions. In what follows, the matrix $J A(t)$ involved in system (1.5) will be called, for simplicity, Hamiltonian; in literature, other terms are also used, for instance, infinitesimal symplectic matrix.

The multipliers of system (1.5) are the eigenvalues of its monodromy matrix $V$, that is, of the matrix $V=X(T)$, where $X(t)$ is a fundamental matrix of solutions (FMS) of system (1.5). As a FMS of system (1.5), we consider the solution to the matrix Cauchy problem: $X^{\prime}=J A(t) X$, $X(0)=I$; here $I$ is the identity matrix of dimension $2 N$.

The following facts hold:

- Let LPHS 1.5 possesses a multiplier $\mu_{0}$. Then $\mu_{0} \neq 0$ and the numbers $\frac{1}{\mu_{0}}, \overline{\mu_{0}}, \frac{1}{\bar{\mu}_{0}}$ are also the multipliers of LPHS (1.5) and they are of the same algebraic and geometric multiplicity and are of the same index.

- If LPHS (1.5) has a multiplicator 1 (or -1), then this multiplier has an even algebraic multiplicity.

- LPHS (1.5) is stable if and only if all its multipliers $\mu$ are located on the unit circumference, that is, $|\mu|=1$, and are semi-simple. At that, LPHS $(1.5)$ is not necessarily asymptotically stable.

Together with (1.5), we shall also consider a perturbed LPHS of form:

$$
\frac{d x}{d t}=J \tilde{A}(t) x, \quad x \in \mathbb{R}^{2 N},
$$

in which $\tilde{A}(t)$ is a small perturbation of the matrix $A(t)$ in the class of symmetric, continuous and $T$-periodic matrices.

Let all multipliers $\mu$ of system $(1.5)$ be located on the unit circle, that is, $|\mu|=1$, and be simple. Let $\mu_{0}$ be one of these multipliers. Then for small perturbations system (1.6) also has a simple multiplier $\tilde{\mu}$ close to $\mu_{0}$ and $|\tilde{\mu}|=1$. The stability property of system $(1.5)$ in this case do not change: it remains stable.

Now let system (1.5) has a multiple multiplier $\mu_{0}$ such that $\left|\mu_{0}\right|=1$ and other multipliers are still simple and are located on the unit circumference. For simplicity, let this multiplicity be 2 . Then while passing from $(1.5)$ to a perturbed system $(1.6)$, the multiplier $\mu_{0}$ usually splits into two simple multipliers $\mu_{1}$ and $\mu_{2}$. At that, two cases are possible: 


$$
\begin{aligned}
& \text { a) } \mu_{2}=1 / \overline{\mu_{1}}: \quad\left|\mu_{1}\right|<1<\left|\mu_{2}\right| \\
& \text { b) }\left|\mu_{1}\right|=\left|\mu_{2}\right|=1 .
\end{aligned}
$$

In Case a), perturbed system (1.6) becomes unstable, while in Case b) it is stable.

Rigorously speaking, the question on which of Cases a) or b) is chosen by system (1.5) under the splitting of a multiple multiplier $\mu_{0}$ depends not only on the properties of perturbed system (1.6) but also on unperturbed system (1.5). Namely, multiple multipliers are divided into two types: definite and indefinite, see, for instance, [2]. If a multiple multiplier $\mu_{0}$ is definite, then for each sufficiently small linear periodic Hamiltonian perturbation of system (1.5) the multiplier $\mu_{0}$ can split only via Case b), that is, the multiplier $\mu_{1}$ and $\mu_{2}$ stay on the unit circumference. If the multiple multiplier $\mu_{0}$ is indefinite, then there exist small perturbations which move these multipliers away from the unit circumference.

The notion of the definiteness of the multipliers is related with another important notion of the theory of LPHS. We say, see, for instance, [2], [5], that system (1.5) is strongly (parametrically) stable if it and all its small linear periodic Hamiltonian perturbations are stable in the Lyapunov sense. In other words, system (1.5) is strongly (parametrically) stable if this system and close to it perturbed systems $(1.6)$ are stable.

The following statement is of an important value, see, for instance, [2], [5].

Theorem 1.1 (Krein-Gelfand-Lidskii). System (1.5) is strongly stable if and only if

1) all its multipliers are semi-simple and their absolute values are equal to one;

2) the numbers \pm 1 are not its multipliers;

3) all its multiple multipliers are definite.

\section{Formulation of PROBlem}

We return back to main systems (1.1) and (1.4).

For unperturbed autonomous system (1.4), the monodromy matrix $V$ in the $T$-periodic problem reads as $V=e^{T J A_{0}}$. The multipliers $\mu$ of system (1.4) are related with the eigenvalues $\lambda$ of the matrix $J A_{0}$ by the identity $\mu=e^{T \lambda}$. By the aforementioned properties of linear Hamiltonian system and in accordance with the perturbation theory of linear operators, see, for instance, [19]), the following holds: if the matrix $J A_{0}$ possesses at least one eigenvalue with a non-zero real part, then perturbed LPHS (1.1) is unstable for all small $|\varepsilon|$.

Let all eigenvalues of the matrix $J A_{0}$ be pure imaginary, namely, these are numbers

$$
\pm i \omega_{1}, \quad \pm i \omega_{2}, \quad \ldots, \quad \pm i \omega_{N},
$$

where $\omega_{j} \geqslant 0$. If the algebraic multiplicity of some eigenvalue $i \omega_{m}$ is $k$, then in list (2.1) the number $i \omega_{m}$ appears exactly $k$ times. In this case, as $\varepsilon=0$, the absolute values of all multipliers of LPHS (1.1) are equal to one. As it has been mentioned above, the situations, when some of these multipliers are multiple, are of a special interest.

Multiple multipliers of system (1.4) arise under one of the following condition, see, for instance [1]):

S1) among number (2.1), there exists at least one $i \omega_{m_{0}}$ such that

$$
\omega_{m_{0}}=\frac{\pi k_{0}}{T} \text { for some integer non-negative } k_{0} ;
$$

S2) among numbers (2.1) there exists at least one pair $i \omega_{m_{0}}$ and $i \omega_{l_{0}}\left(m_{0} \neq l_{0}\right)$ such that

$$
\omega_{m_{0}}-\omega_{l_{0}}=\frac{2 \pi k_{0}}{T} \text { for some integer } k_{0} .
$$


Remark 2.1. Identity (2.2) means that the corresponding multiplier of system (1.4) is equal to 1 if $k_{0}$ is even or -1 if $k_{0}$ is odd and this multiplier is of an even multiplicity. By KreinGelfand-Lidskii theorem this implies that under identity (2.2) unperturbed system (1.4) does not possess the property of strong stability.

Remark 2.2. Identity (2.3) means that the corresponding multiplier of system (1.4) is multiple and it equals to

$$
\mu_{0}=e^{T \omega_{m_{0}} i}=e^{T \omega_{l_{0}}} .
$$

If at the same time the numbers $\omega_{m_{0}}$ and $\omega_{l_{0}}$ do not satisfy relations of form (2.2), that is, $\omega_{m_{0}}, \omega_{l_{0}} \neq \pi k / T$ for all integerl $k$, then multiplier (2.4) obeys the inequality $\mu_{0} \neq \pm 1$.

Condition $\mathbf{S} 2$ covers also the case when the matrix $J A_{0}$ has a multiple pure imaginary eigenvalue. Namely, this is the case if identity 2.3) holds with $k_{0}=0$ : then $\omega_{m_{0}} i=\omega_{l_{0}} i$.

The problem on stability of system (1.1) under conditions of type $\mathbf{S 1}$ or $\mathbf{S} 2$ is often called problem on parametric resonance, see, for instance, 1 1, [7, and the relations are called parametric resonances. Relation (2.2) is called a simple resonance, while a relation of type $(2.3)$ is called combinational resonance. In view of this we mention that relations (2.2) and (2.3) can be represented in an universal form:

$$
n_{1} \omega_{m_{0}}+n_{2} \omega_{l_{0}}=\frac{2 \pi k_{0}}{T},
$$

in which $n_{1}$ and $n_{2}$ are integer numbers such that $\left|n_{1}\right|+\left|n_{2}\right|=2$. In this case one says about a second order resonance.

A lot of works are devoted to studying the stability of linear Hamiltonian systems with a periodic perturbation, in particular, to the problem on parametric resonance. The most part of studies are based on renormalization methods of linear Hamiltonian systems and on a transformation of the Hamiltonian of system (1.1) by means of a canonical change of variables. In this direction, a series of important results was obtained, see, for instance, [1], [11]-[17].

Other approaches to studying the problem on parametric resonance are based on the classical perturbation theory of linear operators. It should be said that direct application of the methods of general theory is not of interest since this way, as a rule, is extremely cumbersome and therefore is not applied in practice. At the same, the main interest is related with the modifications of these methods, which as much as possible take into consideration the features of the problem associated with the Hamiltonian property of the systems. This approach was developed in works by many authors, see, for instance, [2], [5] - [8].

Nowadays the researches are continued in various directions. It is most topical to develop general approaches for investigating the problem on parametric resonance in terms of the original equations without the need of preliminary transformation, which is often laborious and cumbersome. The main difficulty is the problem of constructing formulae for the first correctors of the multipliers of a perturbed non-autonomous periodic Hamiltonian system. In this regard, we point out that the formulae known in the literature, as a rule, are aimed at studying autonomous systems, see, for instance, [5], 8], [10], [18].

In the present paper we study the problem on parametric resonance for LPHS (1.1) under Conditions S1 and S2. We provide new formulae for the first correctors while approximately constructing the multipliers for system (1.1) taking into consideration their definiteness or indefiniteness. The obtained formula are used for analyzing the stability of LPHS (1.1) in the Lyapunov sense.

The problem on parametric resonance of LPHS (1.1) is studied in the following main cases corresponding to conditions $\mathbf{S} 1$ and $\mathbf{S 2}$ :

$P_{1}$. The matrix $J A_{0}$ has a multiple (double) eigenvalue $\lambda=i \omega_{0}$, where $\omega_{0} \geqslant 0$ and $\omega_{0} \neq \pi k / T$ for natural $k$. 
$P_{2}$. The matrix $J A_{0}$ possesses two simple eigenvalues $\lambda_{1}=i \omega_{1}$ and $\lambda_{2}=i \omega_{2}$, where $\omega_{1}, \omega_{2}>0$, $\omega_{1}, \omega_{2} \neq \pi k / T$ for natural $k$ and $\omega_{1}-\omega_{2}=2 \pi k_{0} / T$ for some natural $k_{0}$.

$P_{3}$. The matrix $J A_{0}$ has a simple eigenvalue $\lambda=i \omega_{0}$, where $\omega_{0}=\pi k_{0} / T$ for some natural $k_{0}$.

We assume that the other eigenvalues $\lambda$ of the matrix $J A_{0}$, different from $\pm i \omega_{0}$ in Cases $P_{1}$ and $P_{3}$ and from $\pm i \omega_{1}$ and $\pm i \omega_{2}$ in Case $P_{2}$, are simple and pure imaginary, namely, they are of form $\lambda=i \omega$, where $\omega \neq \pi k / T$ for integer $k$. At the same time, none of these pairs satisfies a resonance relation like one given in Case $P_{2}$.

Cases $P_{1}$ and $P_{2}$ correspond to condition (2.3), while Case $P_{3}$ does to condition (2.2). However, if in Case $P_{1}$ we have $\omega_{0}=0$, then it corresponds to both conditions $(2.2)$ and (2.3).

It is convenient to represent system (1.1) in the form

$$
\frac{d x}{d t}=J\left[A_{0}+\varepsilon S_{1}(t)+S_{2}(t, \varepsilon)\right] x, \quad x \in \mathbb{R}^{2 N},
$$

where $J$ is matrix (1.2), $A_{0}$ is matrix $(1.3), S_{1}(t)$ and $S_{2}(t, \varepsilon)$ are real symmetric and $T$-periodic in $t$ matrices, and $S_{2}(t, \varepsilon)$ is smooth in $\varepsilon$ and satisfies the relation $\left\|S_{2}(t, \varepsilon)\right\|=O\left(\varepsilon^{2}\right)$ as $\varepsilon \rightarrow 0$ uniformly in $t$.

\section{CASE $P_{1}$}

We begin studying of problem from Case $P_{1}$. This case is partitioned into subcases, when the eigenvalue $\lambda=i \omega_{0}$ is semi-simple (subcase $P_{1}^{1}$ ) or not (subcase $P_{1}^{2}$ ). We proceed to considering these subcases.

3.1. Subcase $P_{1}^{1}$. Let the matrix $J A_{0}$ has a semi-simple (double) eigenvalue $i \omega_{0}$, where $\omega_{0} \geqslant 0$ and $\omega_{0} \neq \pi k / T$ for natural $k$. We denote by $V(\varepsilon)$ the monodromy matrix of perturbed system 2.5). Then $V_{0}=e^{J A_{0} T}$ is the monodromy matrix of unperturbed matrix (1.4). In the considered subcase the matrix $V_{0}$ has a semi-simple eigenvalue $\mu_{0}=e^{i \omega_{0} T}$ of multiplicity 2 . We note that since $\omega_{0} \neq \pi k / T$ for natural $k$, then for $\omega_{0}>0$ we have the inequality $\mu_{0} \neq \pm 1$. We also note that as $\omega_{0}>0$ the multiplier $\mu_{0}$ of system (1.4) can be both definite and indefinite, while as $\omega_{0}=0$, it is indefinite.

Namely, as $\omega_{0}>0$, two mutually exclusive cases are possible, see, for instance, [2], [5]:

$1^{0}$ for each eigenvector $e$ associated with the eigenvalue $i \omega_{0}$ of the matrix $J A_{0}$ the relation holds: $(J e, e) \neq 0$;

$2^{0}$ there exists an eigenvector $e$ associated with the eigenvalue $i \omega_{0}$ of the matrix $J A_{0}$, for which $(J e, e)=0$.

In the first case the mutliplier $\mu_{0}=e^{i \omega_{0} T}$ of system 1.4 is definite, while in the second case it is indefinite.

Since the matrix $J A_{0}$ has a semi-simple (double) eigenvalue $i \omega_{0}$, then there exist non-zero linearly independent vectors $e, g \in \mathbb{C}^{2 N}$ such that

$$
J A_{0} e=i \omega_{0} e, \quad J A_{0} g=i \omega_{0} g .
$$

The vectors $e, g$ are the eigenvectors also for the monodromy matrix $V_{0}=e^{J A_{0} T}$ and are associated with a semi-simple eigenvalue $\mu_{0}=e^{i \omega_{0} T}$ of multiplicity 2 .

The aforementioned properties of the multipliers $\mu_{0}$ related with the definiteness and indefinitenss imply the following statement.

Lemma 3.1. Let $\mu_{0}$ be a definite multiplier of system (1.4). Then the eigenvectors e, $g$ of the matrix $J A_{0}$ can be normalized only by one of the following identities:

$$
(i J e, e)=(i J g, g)=1
$$

or

$$
(i J e, e)=(i J g, g)=-1
$$


If $\mu_{0}$ is an indefinite multiplier, then the vectors e, $g$ can be normalized by the identities

$$
(i J e, e)=-1, \quad(i J g, g)=1 .
$$

Here the vectors $e, g$ can be chosen satisfying the identity:

$$
(e, J g)=0 \text {. }
$$

According to the perturbation theory of linear operators, see, for instance, [19]), for small $|\varepsilon|$ the matrix $V(\varepsilon)$ has a pair of eigenvalues $\mu_{1}(\varepsilon)$ and $\mu_{2}(\varepsilon)$ such that the functions $\mu_{1}(\varepsilon)$ and $\mu_{2}(\varepsilon)$ are continuously differentiable, and $\mu_{1}(0)=\mu_{2}(0)=\mu_{0}$. Moreover, they can be represented in the form

$$
\mu_{1}(\varepsilon)=\mu_{0}+\mu_{1}^{(1)} \varepsilon+O\left(\varepsilon^{3 / 2}\right), \quad \mu_{2}(\varepsilon)=\mu_{0}+\mu_{1}^{(2)} \varepsilon+O\left(\varepsilon^{3 / 2}\right) .
$$

Let us provide the scheme of constructing the coefficients $\mu_{1}^{(1)}$ and $\mu_{1}^{(2)}$ in formulae 3.6 .

Let the vectors $e, g$ be normalized in accordance with Lemma 3.1. We define a constant matrix

$$
S_{0}=\int_{0}^{T} S_{1}(t) d t
$$

where $S_{1}(t)$ is a matrix involved in system 2.5$)$. We let

$$
a=\left(S_{0} e, e\right), \quad b=\left(S_{0} g, g\right), \quad c=\left(S_{0} g, e\right) .
$$

We note that the numbers $a$ and $b$ are real, while the number $c$ is in general complex.

3.1.1. Perturbation of definite mulitplier. We first consider the coefficients $\mu_{1}^{(1)}$ and $\mu_{1}^{(2)}$ in formulae (3.6) under the assumption that $\mu_{0}=e^{i \omega_{0} T}$ is a definite multiplier of system (1.4). We note that then $\mu_{0} \neq \pm 1$ and therefore, in formula $\mu_{0}=e^{i \omega_{0} T}$ we necessarily have $\omega_{0} \neq \pi k / T$ for integer $k$. In particular, here we should assume that $\omega_{0} \neq 0$.

Theorem 3.1. Let one of normalizations (3.2) and (3.3) hold and identity (3.5) be satisfied. Then the coefficients $\mu_{1}^{(1)}$ and $\mu_{1}^{(2)}$ in expansions (3.6) are the eigenvalues of the matrices $D=$ $-i \mu_{0} D_{0}$ and $D=i \mu_{0} D_{0}$ respectively, where

$$
D_{0}=\left[\begin{array}{ll}
a & c \\
\bar{c} & b
\end{array}\right] .
$$

The proof of this and other main statements of the paper are given in Section 7 .

We note that the eigenvalues $\lambda_{1}$ and $\lambda_{2}$ of matrix (3.9) are real numbers, namely, they are roots of the quadratic equation

$$
\lambda^{2}-(a+b) \lambda+a b-c \bar{c}=0 .
$$

We provide some corollaries from Theorem 3.1 .

Corollary 3.1. Let $\mu_{0}$ be a definite multiplier of system (1.4). Let normalization (3.2) or normalization (3.3) hold. Then the coefficients $\mu_{1}^{(1)}$ and $\mu_{1}^{(2)}$ in expansions (3.6) read as

$$
\mu_{1}^{(1)}=-i \mu_{0} \lambda_{1}, \quad \mu_{1}^{(2)}=-i \mu_{0} \lambda_{2} \quad\left(\mu_{1}^{(1)}=i \mu_{0} \lambda_{1}, \quad \mu_{1}^{(2)}=i \mu_{0} \lambda_{2}\right),
$$

where $\lambda_{1}$ and $\lambda_{2}$ are the roots of quadratic equation (3.10).

Corollary 3.2. Let $\mu_{0}$ be a definite multiplier of system (1.4) and normalization (3.2) or (3.3) hold. Then for $\omega_{0}>0$ unperturbed system (1.4) is strongly stable. While passing to perturbed system (1.1), the definite multiplier $\mu_{0}$ splits in accordance with formulae (3.6) and (3.11) staying on the unit circumference: $\left|\mu_{1}(\varepsilon)\right|=\left|\mu_{2}(\varepsilon)\right|=1$. For small $|\varepsilon|$ system (1.1) is stable. 
3.1.2. Perturbation of indefinite multiplier. Now let $\mu_{0}=e^{i \omega_{0} T}$ be an indefinite multiplier of system (1.4).

Theorem 3.2. Let normalization (3.4) and identity 3.5 hold. Then the coefficients $\mu_{1}^{(1)}$ and $\mu_{1}^{(2)}$ in expansions (3.6) are the eigenvalues of the matrix $D=i \mu_{0} D_{1}$, where

$$
D_{1}=\left[\begin{array}{cc}
a & c \\
-\bar{c} & -b
\end{array}\right] \text {. }
$$

The eigenvalues $\lambda_{1}$ and $\lambda_{2}$ of the matrix 3.12 are the roots of the quadratic equation

$$
\lambda^{2}+(a-b) \lambda-a b+c \bar{c}=0
$$

and therefore, they can be both real and complex. We denote by $\Delta=(a+b)^{2}-4 c \bar{c}$ the discriminant of equation (3.13).

We provide corollaries from Theorem 3.2 .

Corollary 3.3. Let $\mu_{0}$ be an indefinite multiplier of system (1.4) and normalization (3.4) hold. Then the coefficients $\mu_{1}^{(1)}$ and $\mu_{1}^{(2)}$ in expansions (3.6) read as

$$
\mu_{1}^{(1)}=-i \mu_{0} \lambda_{1}, \quad \mu_{1}^{(2)}=-i \mu_{0} \lambda_{2}
$$

where $\lambda_{1}$ and $\lambda_{2}$ are the roots of quadratic equation (3.13). For small $|\varepsilon|$, the indefinite multiplier $\mu_{0}$ of system (1.4) splits according to formulae (3.6) and (3.14).

Corollary 3.4. Let $\Delta>0$. Then for a given perturbation $S_{1}(t)$ of system (2.5) and all small $|\varepsilon|$ the indefinite mutliplier $\mu_{0}$ of system (1.4) splits but stays on the unit circle: $\left|\mu_{1}(\varepsilon)\right|=$ $\left|\mu_{2}(\varepsilon)\right|=1$. For small $|\varepsilon|$ system $(2.5)$ is stable.

Corollary 3.5. Let $\Delta<0$. Then for a given perturbation $S_{1}(t)$ of system (2.5) and all small non-zero $|\varepsilon|$ the indefinite multiplier $\mu_{0}$ of system (1.4) splits leaving the unit circle: $\left|\mu_{1}(\varepsilon)\right|<1$ and $\left|\mu_{2}(\varepsilon)\right|>1$. For small non-zero $|\varepsilon|$ system (2.5) is unstable.

3.2. Subcase $P_{1}^{2}$. Let the matrix $J A_{0}$ possesses a non-semi-simple (double) eigenvalue $i \omega_{0}$, where $\omega_{0} \geqslant 0$ and $\omega_{0} \neq \pi k / T$ for natural $k$. Then the monodromy matrix $V_{0}$ of unperturbed system (1.4) has a non-semi-simple eigenvalue $\mu_{0}=e^{\omega_{0} T i}$ of multiplicity 2 . According to the perturbation theory of linear operator, for small $|\varepsilon|$ the monodromy matrix $V(\varepsilon)$ of perturbed system (2.5) has a pair of eigenvalues $\mu_{1}(\varepsilon)$ and $\mu_{2}(\varepsilon)$ such that the functions $\mu_{1}(\varepsilon)$ and $\mu_{2}(\varepsilon)$ are continuous and $\mu_{1}(0)=\mu_{2}(0)=\mu_{0}$. Moreover, they can be represented by Puiseux series:

$$
\mu_{1}(\varepsilon)=\mu_{0}+\mu_{1}^{(1)} \varepsilon^{1 / 2}+O(\varepsilon), \quad \mu_{2}(\varepsilon)=\mu_{0}+\mu_{2}^{(1)} \varepsilon^{1 / 2}+O(\varepsilon) .
$$

We proceed to calculating the coefficients $\mu_{1}^{(j)}$ in formulae 3.15 . We observe that in the considered case there exists a pair of non-zero linearly independent vectors $e, g \in \mathbb{C}^{2 N}$ such that the identities hold:

$$
J A_{0} e=i \omega_{0} e, \quad J A_{0} g=i \omega_{0} g+e .
$$

We also mention the identities $V_{0} e=\mu_{0} e$ and $V_{0} g=\mu_{0}(g+T e)$.

It is easy to prove the following statement.

Lemma 3.2. The relations $(e, J e)=0,(e, J g) \neq 0$ hold and the number $(e, J g)$ is real. The vector $g$ can be chosen by the identity

$$
(g, J g)=0 .
$$

In what follows we suppose that identity 3.17 holds. By Lemma 3.2 the number $\nu=\frac{1}{(e, J g)}$ is well-defined. 
Theorem 3.3. The coefficients $\mu_{1}^{(1)}$ and $\mu_{2}^{(1)}$ in expansions 3.15) read as

$$
\mu_{1}^{(1)}=\mu_{0} \sqrt{-T \nu\left(S_{0} e, e\right)}, \quad \mu_{2}^{(1)}=-\mu_{1}^{(1)}
$$

where $S_{0}$ is matrix (3.7).

We note that the radicand in formula $(3.18)$ is real.

We provide some corollaries from Theorem 3.3 .

Corollary 3.6. In Subcase $P_{1}^{2}$ unperturbed system (1.4) is not strongly stable and respectively, its multiplier $\mu_{0}=e^{i \omega_{0} T}$ is indefinite. For small $|\varepsilon|$ the multiplier $\mu_{0}$ splits in accordance with formulae (3.15) and (3.18).

Corollary 3.7. Let $\varepsilon \nu\left(S_{0} e, e\right)>0$. Then for a given perturbation $S_{1}(t)$ of system (2.5), for small $|\varepsilon|$, the multiplier $\mu_{0}$ of system (1.4) stays on the unit circumference: $\left|\mu_{1}(\varepsilon)\right|=\left|\mu_{2}(\varepsilon)\right|=$ 1. In this case system (2.5) is stable.

Corollary 3.8. Let $\varepsilon \nu\left(S_{0} e, e\right)<0$. Then for a given perturbation $S_{1}(t)$ of system (2.5) for corresponding small $|\varepsilon|$, the multiplier $\mu_{0}$ of system (1.4) leaves the unit circumference $\left|\mu_{1}(\varepsilon)\right|<1$ and $\left|\mu_{2}(\varepsilon)\right|>1$. In this case system (2.5) is unstable.

3.3. Case of zero eigenvalue. An important particular version of Case $P_{1}$ is a situation, when the matrix $J A_{0}$ has a zero eigenvalue $\lambda=0$ of multiplicity 2 . Here there are some features.

We first mention that in this situation in formulae (3.6) and (3.15) we have $\mu_{0}=1$. By Krein-Gelfand-Lidskii this implies that then system (1.4) does not possesses the property of strong stability.

Second, the eigenvalue $\lambda=0$ can be semi-simple or not. This is this situation will be studied in accordance with the formulae and conclusions of Subsections 3.1 .2 and 3.2 .

\section{CASE $P_{2}$}

Here we consider problem on parametric resonance $(2.5)$ in Case $P_{2}$, that is, as $J A_{0}$ two simple eigenvalues $\lambda_{1}=i \omega_{1}$ and $\lambda_{2}=i \omega_{2}$, where $\omega_{1}, \omega_{2}>0, \omega_{1}, \omega_{2} \neq \pi k / T$ for natural $k$ and $\omega_{1}-\omega_{2}=2 \pi k_{0} / T$ for some natural $k_{0}$. Let, as above, $V(\varepsilon)$ is the monodromy matrix of perturbed system $(2.5)$. Then the monodromy matrix $V_{0}=e^{J A_{0} T}$ of unperturbed system (1.4) has a semi-simple eigenvalue $\mu_{0}$ of multiplicity 2 , namely, $\mu_{0}=e^{T \omega_{1} i}=e^{T \omega_{2} i}$. At that, since $\omega_{1}, \omega_{2} \neq \pi k / T$ for natural $k$, we have $\mu_{0} \neq \pm 1$.

For small $|\varepsilon|$ the matrix $V(\varepsilon)$ has two eigenvalues $\mu_{1}(\varepsilon)$ and $\mu_{2}(\varepsilon)$ such that the functions $\mu_{1}(\varepsilon)$ and $\mu_{2}(\varepsilon)$ are continuously differentiable and can be represented in form (3.6).

Since in considered Case $P_{2}$ the multiplier $\mu_{0}$ of system (1.4) is semi-simple, the coefficients $\mu_{1}^{(1)}$ and $\mu_{1}^{(2)}$ in formulae 3.6 can be constructed by the same scheme as for Cubcase $P_{1}^{1}$, see Subsection 3.1. Here we mention just some features related to Case $P_{2}$.

First, instead of identities $(3.1)$, which hold in subcase $P_{1}^{1}$, in Case $P_{2}$ there exists a pair of linearly independent vectors $e, g \in \mathbb{C}^{2 N}$ such that the identities

$$
J A_{0} e=i \omega_{1} e, \quad J A_{0} g=i \omega_{2} g
$$

are true. These vectors are also eigenvectors of the monodromy matrix $V_{0}=e^{J A_{0} T}$ associated with the semi-simple double eigenvalue $\mu_{0}$.

Second, although here a complete analogue of Lemma 3.1 holds true, the proof of this analogue has its own features. In particular, there is no need to ensure identity (3.5) since now it is always true. 
Finally, third, instead of numbers $(3.8)$ in matrices $(3.9)$ and $(3.12)$ in Case $P_{2}$ we should employ the numbers

$$
a=\left(S_{0} e, e\right), \quad b=\left(S_{0} g, g\right), \quad c=\int_{0}^{T} e^{-2 \pi i k_{0} t / T}\left(S_{1}(t) g, e\right) d t
$$

here $S_{0}$ is matrix (3.7).

In view of the mentioned features in the considered Case $P_{2}$, complete analogues of Theorems 3.1 and 3.2 hold as well as of their corollaries.

We note that as $k_{0}=0$, the formulae for Case $P_{2}$ coincide with the corresponding formulae of the above considered Subcase $P_{1}^{1}$. This is natural since the identity $k_{0}=0$ means that $\omega_{1}=\omega_{2}$, that is, the matrix $J A_{0}$ has a multiple semi-simple eigenvalue $\lambda=i \omega_{0}$; here $\omega_{0}=\omega_{1}=\omega_{2}$.

\section{CASE $P_{3}$}

We proceed to Case $P_{3}$. Let the matrix $J A_{0}$ has a simple eigenvalue $i \omega_{0}$, where $\omega_{0}=\pi k_{0} / T$ for some natural $k_{0}$. Then the monodromy matrix $V_{0}=e^{J A_{0} T}$ of unperturbed system (1.4) has a semi-simple eigenvalue $\mu_{0}$ of multiplicity 2 , where $\mu_{0}=1$ if $k_{0}$ is even and $\mu_{0}=-1$ if $k_{0}$ is odd. It follows from Krein-Gelfand-Lidskii theorem that in the considered case system (1.4) does not possess the property of strong stability.

For small $|\varepsilon|$, the monodromy matrix $V(\varepsilon)$ of perturbed system (2.5) possesses a pair of eigenvalues $\mu_{1}(\varepsilon)$ such that $\mu_{2}(\varepsilon) \mu_{1}(0)=\mu_{2}(0)=\mu_{0}$. The functions $\mu_{1}(\varepsilon)$ and $\mu_{2}(\varepsilon)$ are continuously differentiable and are represented in form (3.6).

Let us provide a statement about calculating the coefficients $\mu_{1}^{(j)}$ in formulae $(3.6)$. We mention that in the considered case there exists a non-zero vector $e+i g \in \mathbb{C}^{2 N}$, where $e, g \in \mathbb{R}^{2 N}$ such that

$$
J A_{0}(e+i g)=i \omega_{0}(e+i g) .
$$

The vectors $e, g \in \mathbb{R}^{2 N}$ turn out to be eigenvectors also for the monodromy matrix $V_{0}=e^{J A_{0} T}$ associated with a semi-simple eigenvalue $\mu_{0}$ of multiplicity 2 .

Lemma 5.1. The inequality $(e, J g) \neq 0$ holds.

We let

$$
\nu=\frac{1}{(e, J g)} .
$$

The number $(e, J g)$, and therefore, the number $\nu$ are real. We define a matrix:

$$
B=\nu \mu_{0}\left[\begin{array}{cc}
a & b_{1} \\
b_{2} & -a
\end{array}\right],
$$

in which the numbers $a, b_{1}$ and $b_{2}$ are given by the identities:

$$
\begin{aligned}
& a=\int_{0}^{T}\left\{\cos \left(2 \omega_{0} t\right)\left(S_{1}(t) e, g\right)-\frac{1}{2} \sin \left(2 \omega_{0} t\right)\left[\left(S_{1}(t) g, g\right)-\left(S_{1}(t) e, e\right)\right]\right\} d t, \\
& b_{1}=\int_{0}^{T}\left[\cos ^{2}\left(\omega_{0} t\right)\left(S_{1}(t) g, g\right)+\sin ^{2}\left(\omega_{0} t\right)\left(S_{1}(t) e, e\right)+\sin \left(2 \omega_{0} t\right)\left(S_{1}(t) e, g\right)\right] d t, \\
& b_{2}=b_{1}-\left[\left(S_{0} e, e\right)+\left(S_{0} g, g\right)\right] ;
\end{aligned}
$$

here $S_{0}$ is matrix 3.7 .

Theorem 5.1. The coefficients $\mu_{1}^{(1)}$ and $\mu_{1}^{(2)}$ in formulae (3.6) are the eigenvalues of matrix (5.3). 
We let

$$
\Delta=a^{2}+b_{1} b_{2}
$$

The eigenvalues $\lambda_{1}$ and $\lambda_{2}$ of matrix (5.3) are numbers $\lambda_{1,2}= \pm \nu \mu_{0} \sqrt{\Delta}$, which can be both real and pure imaginary. Therefore, the coefficients $\mu_{1}^{(1)}$ and $\mu_{1}^{(2)}$ in formulae (3.6) are the numbers

$$
\mu_{1}^{(1)}=\nu \mu_{0} \sqrt{\Delta}, \quad \mu_{1}^{(2)}=-\mu_{1}^{(1)} .
$$

We provide some corollaries from Theorem 5.1 .

Corollary 5.1. In Case $P_{3}$ the multiplier $\mu_{0}$ of system (1.4) is equal to $\mu_{0}=1$ or $\mu_{0}=-1$ and is semi-simple of multiplicity 2. This multiplier is indefinite and unperturbed system (1.4) is not strongly stable. For small $|\varepsilon|$ the multiplier $\mu_{0}$ splits in accordance with formulae (3.6) and (5.8).

Corollary 5.2. Let $\Delta<0$. Then for a given perturbation $S_{1}(t)$ of system (2.5) for small $|\varepsilon|$ the multiplier $\mu_{0}$ of system (1.4) stays on the unit circumference: $\left|\mu_{1}(\varepsilon)\right|=\left|\mu_{2}(\varepsilon)\right|=1$. In this case system (2.5) remains stable.

Corollary 5.3. Let $\Delta>0$. Then for a given perturbation $S_{1}(t)$ of system (2.5) for small non-zero $|\varepsilon|$ the multiplier $\mu_{0}$ of system (1.4) leaves the unit circumference: $\left|\mu_{1}(\varepsilon)\right|<1$ and $\left|\mu_{2}(\varepsilon)\right|>1$. In this case for small non-zero $|\varepsilon|$ system (2.5) is unstable.

\section{APPENDIX: STABILITY OF LIBRATION POINTS IN THREE BODIES PROBLEM}

6.1. Formulation of problem. As an application we consider the stability of triangle libration points in a planar bounded elliptic three-bodies problem (PBETBP), see, for instance, [5], [11]. This problem in the linear setting gives rise to a system of differential equations:

$$
\frac{d x}{d t}=J A(t, \varepsilon, \mu) x, \quad x \in \mathbb{R}^{4},
$$

in which $J$ is matrix 1.2 of order $4 \times 4, A(t, \varepsilon, \mu)$ is a symmetric matrix:

$$
A(t, \varepsilon, \mu)=\left[\begin{array}{cccc}
1-\frac{3}{4} \rho(t, \varepsilon) & \frac{3 \sqrt{3}}{4}(2 \mu-1) \rho(t, \varepsilon) & 0 & -1 \\
\frac{3 \sqrt{3}}{4}(2 \mu-1) \rho(t, \varepsilon) & 1-\frac{9}{4} \rho(t, \varepsilon) & 1 & 0 \\
0 & 1 & 1 & 0 \\
-1 & 0 & 0 & 1
\end{array}\right] .
$$

Here $\rho(t, \varepsilon)=(1+\varepsilon \cos t)^{-1}, \varepsilon$ is the eccentricity of Kepler orbit $(0 \leqslant \varepsilon<1)$, $\mu$ is a mass parameter $(0<\mu<1)$. System (6.1) is a linear periodic (with a period $T=2 \pi$ ) Hamiltonian system.

Numerous studies are devoted to the stability of triangle libration points in PBETBP. One of the most interesting issues is that on constructing the stability domains for system (6.1) in the plane of parameters $(\mu, \varepsilon)$. The main known results are presented in monograph [11]. The researches in this directions are still active, see, for instance, [22]-[25].

On Figure 1, we draw the stability and instability domains for system (6.1) for small values of $\mu$.

The crosshatched region corresponds to the stability. The boundary of the domain is formed by three continuous curves $\Gamma_{1}, \Gamma_{2}$ and $\Gamma_{3}$. These curves approach the axis $\mu$ at the points

$$
\mu_{0}=\frac{1}{2}-\frac{\sqrt{2}}{3}=0,028595 \ldots, \quad \mu^{*}=\frac{1}{2}-\frac{\sqrt{69}}{18}=0,038520 \ldots .
$$

In this section, as an illustration of the above results, we discuss the construction of tangentials to the curves $\Gamma_{1}, \Gamma_{2}$ and $\Gamma_{3}$ at the points $\left(\mu_{0}, 0\right)$ and $\left(\mu^{*}, 0\right)$, respectively. 


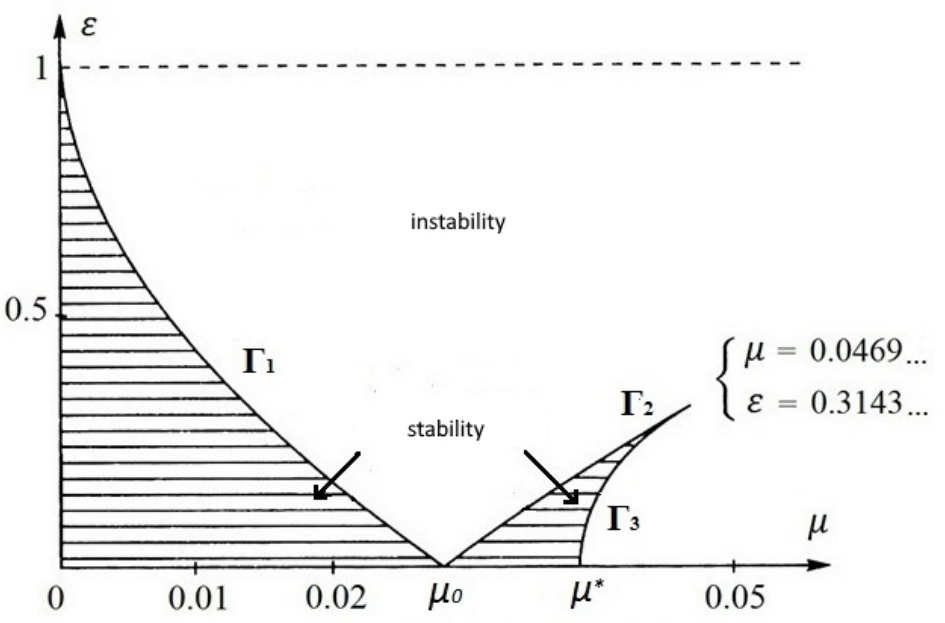

FIGURE 1. Stability domain of triangle libration points

6.2. Auxiliary constructions. As $\varepsilon=0$, system 6.1 is autonomous:

$$
\frac{d x}{d t}=J A_{0}(\mu) x, \quad x \in \mathbb{R}^{4},
$$

where $J A_{0}(\mu)$ is a Hamiltonian matrix:

$$
J A_{0}(\mu)=\left[\begin{array}{cccc}
0 & 1 & 1 & 0 \\
-1 & 0 & 0 & 1 \\
-\frac{1}{4} & \frac{3 \sqrt{3}}{4}(1-2 \mu) & 0 & 1 \\
\frac{3 \sqrt{3}}{4}(1-2 \mu) & \frac{5}{4} & -1 & 0
\end{array}\right] .
$$

An analysis of the characteristic equation of matrix (6.4) shows that

$1^{o}$ the matrix $J A_{0}\left(\mu_{0}\right)$ has a pair of pure imaginary eigenvalues of form $\pm i \omega_{0}$, where $\omega_{0}=$ $1 / 2$. Two other its eigenvalues are the numbers $\pm i \omega_{1}$, where $\omega_{1}=\sqrt{3} / 2$. Thus, as $\mu=\mu_{0}$, condition of type (2.2) holds, or, what is the same, the conditions of Case $P_{3}$, see Subsection 5. As $\mu=\mu_{0}$, system (6.3) has a multiplier $\eta_{0}=e^{i \omega_{0} 2 \pi}=-1$ being semi-simple of multiplicity two and indefinite.

$2^{o}$ the matrix $J A_{0}\left(\mu^{*}\right)$ has a pair of non-semi-simple double pure imaginary eigenvalues of form $\pm i \omega_{0}$, where $\omega_{0}=1 / \sqrt{2}$. Thus, as $\mu=\mu^{*}$, condition of type 2.3 holds, or, what is the same, the conditions of Case $P_{1}$, namely, of Subcase $P_{2}^{1}$, see Subsection 3.2 . As $\mu=\mu^{*}$, system 6.3 has a multiplier $\eta_{0}=e^{i \pi \sqrt{2}}$, which is semi-simple, double and indefinite.

Thus, problem on stability of system (6.1) as $\mu=\mu_{0}$ and as $\mu=\mu^{*}$ for small values of $\varepsilon$ is a problem on parametric resonance. For $\mu=\mu_{0}$, the resonance is simple, while for $\mu=\mu^{*}$ the resonance is combinatorial.

We proceed to studying the stability of system (6.1) under the conditions of Cases $1^{o}$ and $2^{\circ}$.

6.3. Case $1^{\circ}$. We consider the stability of system (6.1) as $\mu$ is close to $\mu_{0}$ and $\varepsilon$ is small. Namely, we study this problem for the values $(\mu, \varepsilon)$ located on the straight line

$$
\mu=\mu_{0}+m \varepsilon,
$$

where $m$ is some fixed coefficient. 
Substituting 6.5 into (6.1) and making appropriate transformations with 6.2 taken into consideration, we obtain the system

$$
\frac{d x}{d t}=J\left[A_{0}+\varepsilon S_{1}(t)+S_{2}(t, \varepsilon)\right] x, \quad x \in \mathbb{R}^{4},
$$

where

$$
\begin{aligned}
& J A_{0}=J A\left(\mu_{0}\right)=\left[\begin{array}{cccc}
0 & 1 & 1 & 0 \\
-1 & 0 & 0 & 1 \\
-\frac{1}{4} & \frac{\sqrt{6}}{2} & 0 & 1 \\
\frac{\sqrt{6}}{2} & \frac{5}{4} & -1 & 0
\end{array}\right], \\
& J S_{1}(t)=-m A_{1}-\cos t A_{2}, \\
& A_{1}=\frac{3 \sqrt{3}}{2}\left[\begin{array}{cccc}
0 & 0 & 0 & 0 \\
0 & 0 & 0 & 0 \\
0 & 1 & 0 & 0 \\
1 & 0 & 0 & 0
\end{array}\right], \quad A_{2}=\left[\begin{array}{cccc}
0 & 0 & 0 & 0 \\
0 & 0 & 0 & 0 \\
\frac{3}{4} & \frac{\sqrt{6}}{2} & 0 & 0 \\
\frac{\sqrt{6}}{2} & \frac{9}{4} & 0 & 0
\end{array}\right],
\end{aligned}
$$

the matrix $S_{2}(t, \varepsilon)$ is symmetric, continuous and $2 \pi$-periodic in $t$, smooth in $\varepsilon$ and satisfies the relation $\left\|S_{2}(t, \varepsilon)\right\|=O\left(\varepsilon^{2}\right)$ as $\varepsilon \rightarrow 0$ uniformly in $t$.

System (6.6) is one of form (2.5). In order to study the stability of system (6.6) we employ Theorem 5.1. First we should construct matrix (5.3). This requires to construct an eigenvector $e+i g$ associated with the eigenvalue $i / 2$ of the matrix $J A_{0}$. As $e$ and $g$ we can take, for instance, the vectors

$$
e=\left[\begin{array}{c}
8 \\
-2 \sqrt{6} \\
3 \sqrt{6} / 2 \\
7
\end{array}\right], \quad g=\left[\begin{array}{c}
\sqrt{6} \\
2 \\
2 \\
0
\end{array}\right]
$$

The numbers $T, \omega_{0}, \mu_{0}$ (which is redenoted here by $\eta_{0}$ ) and $\nu$ involved in formulae (5.3)-(5.6) read as

$$
T=2 \pi, \quad \omega_{0}=\frac{1}{2}, \quad \eta_{0}=-1, \quad \nu=-1 / 7 .
$$

We are in position to calculate the entries of matrix (5.3); after simple calculations we obtain:

$$
B=\frac{\pi}{28}\left[\begin{array}{cc}
-4 \sqrt{6} & 39-504 \sqrt{2} m \\
39+504 \sqrt{2} m & 4 \sqrt{6}
\end{array}\right] .
$$

In particular, number (5.7) here is as follows: $\Delta=49 \pi^{2}\left(33 / 16-648 m^{2}\right)$.

By Corollary 5.1 we then obtain that a multiple multiplier $\eta_{0}=-1$ of system (6.3) with $\mu=\mu_{0}$ splits in accordance with formulae (3.6) and (5.8) while passing to system (6.6). In our case these formulae are of the form:

$$
\eta_{1}(\varepsilon)=-1+\eta_{1}^{(1)} \varepsilon+O\left(\varepsilon^{3 / 2}\right), \quad \eta_{2}(\varepsilon)=-1+\eta_{1}^{(2)} \varepsilon+O\left(\varepsilon^{3 / 2}\right)
$$

here

$$
\eta_{1}^{(1)}=\frac{1}{7} \sqrt{\Delta}, \quad \eta_{1}^{(2)}=-\eta_{1}^{(1)} .
$$

Then by Corollaries 5.2 and 5.3 we obtain that the change of the nature of the stability of system (6.6) occurs as $\Delta=0$, that is, for the values $m$ defined by the identities $m= \pm k_{0}$, 
where

$$
k_{0}=\sqrt{\frac{11}{3456}} .
$$

At that, systemn (6.6) is unstable if $-k_{0}<m<k_{0}$ and it is stable if $m<-k_{0}$ or $m>k_{0}$. This implies that the straight lines

$$
\mu=\mu_{0}+k_{0} \varepsilon, \quad \mu=\mu_{0}-k_{0} \varepsilon
$$

are the sought tangentials to the curves $\Gamma_{1}$ and $\Gamma_{2}$ at the point $\left(\mu_{0}, 0\right)$.

6.4. Case $2^{o}$. We consider the stability of problem 6.1) for $\mu$ close to $\mu^{*}$ and for small $\varepsilon$. Namely, we shall study this problem for the values $(\mu, \varepsilon)$ located on the straight line

$$
\mu=\mu^{*}+m \varepsilon,
$$

where $m$ is some fixed coefficient.

Substituting (6.7) into (6.1) and making corresponding transformations, we obtain system of form (6.6), in which

$$
\begin{aligned}
& J A_{0}=J A\left(\mu^{*}\right)=\left[\begin{array}{cccc}
0 & 1 & 1 & 0 \\
-1 & 0 & 0 & 1 \\
-\frac{1}{4} & \frac{\sqrt{23}}{4} & 0 & 1 \\
\frac{\sqrt{23}}{4} & \frac{5}{4} & -1 & 0
\end{array}\right], \\
& J S_{1}(t)=-m A_{1}-\cos t A_{2}, \\
& A_{1}=\frac{3 \sqrt{3}}{2}\left[\begin{array}{llll}
0 & 0 & 0 & 0 \\
0 & 0 & 0 & 0 \\
0 & 1 & 0 & 0 \\
1 & 0 & 0 & 0
\end{array}\right],
\end{aligned}
$$

In order to study the stability of the obtained problem, we employ Theorem 3.3. We should determine numbers (3.18). In its turn, this requires to construct a pair of non-zero linearly independent vectors $e, g \in \mathbb{C}^{2 N}$ such that identities 3.16 holds as $\omega_{0}=1 / \sqrt{2}$. These vectors and the eigenvector and the adjoint one, respectively, for the monodromy matrix $V_{0}=e^{J A_{0} T}$. They are associated with the double eigenvalue $e^{i \sqrt{2} \pi}$; namely, the identities hold:

$$
V_{0} e=e^{i \sqrt{2} \pi} e, \quad V_{0} g=e^{i \sqrt{2} \pi}(g+T e) .
$$

As $e$ and $g$ we can take, for instance, the vectors

$$
e=\left[\begin{array}{c}
8 \sqrt{2}-2 i \sqrt{23} \\
10 i \\
\sqrt{46}-2 i \\
3 \sqrt{2}-2 i \sqrt{23}
\end{array}\right], \quad g=\frac{1}{3 \sqrt{2}-2 i \sqrt{23}}\left[\begin{array}{c}
-4(9 \sqrt{23}+16 i \sqrt{2}) \\
68-16 i \sqrt{46} \\
-(48+24 i \sqrt{46}) \\
0
\end{array}\right] .
$$

The numbers $T, \omega_{0}, \mu_{0}$ (which is redenoted here by $\eta_{0}$ ) and $\nu$ involved in formulae (3.16) and (3.18) are as follows:

$$
T=2 \pi, \quad \omega_{0}=\frac{1}{\sqrt{2}}, \quad \eta_{0}=e^{i \pi \sqrt{2}}, \quad \nu=\frac{1}{160} .
$$

By Corollary 3.6 this implies that a multiple multiplier $\eta_{0}=e^{i \pi \sqrt{2}}$ of system 6.3 with $\mu=\mu^{*}$ splits according to formulae (3.15) and (3.18) while passing to system (6.6). In our case these formulae read as

$$
\eta_{1}(\varepsilon)=\eta_{0}+\eta_{1}^{(1)} \varepsilon^{1 / 2}+O(\varepsilon), \quad \eta_{2}(\varepsilon)=\eta_{0}+\eta_{1}^{(2)} \varepsilon^{1 / 2}+O(\varepsilon) ;
$$


here

$$
\eta_{1}^{(1)}=\frac{1}{\sqrt{2}} e^{i \pi \sqrt{2}} \sqrt[4]{621} \sqrt{m \pi}, \quad \eta_{2}^{(1)}=-\eta_{1}^{(1)} .
$$

Then by Corollaries 3.7 and 3.8 we obtain that the nature of the stability of system (6.6) changes as $m=0$. Then system (6.6) is unstable (stable) if $m>0(m<0)$. This yields that the vertical straight line $\mu=\mu^{*}$ is the sought tangential to the curve $\Gamma_{3}$ at the point $\left(\mu^{*}, 0\right)$.

\section{Proof of Main Statements}

7.1. Auxiliary constructions. We first recall some auxiliary statements obtained earlier by the authors of the present paper, see [20], [21].

Let $A(\varepsilon)$ be a real square matrix smoothly depending on the parameter $\varepsilon$. First we suppose that the matrix $A_{0}=A(0)$ has a semi-simple double eigenvalue $\lambda_{0}$ (real or complex). Then for small $|\varepsilon|$ the matrix $A(\varepsilon)$ possesses two eigenvalues $\lambda^{(1)}(\varepsilon)$ and $\lambda^{(2)}(\varepsilon)$ such that $\lambda^{(1)}(0)=$ $\lambda^{(2)}(0)=\lambda_{0}$. The mentioned functions are continuously differentiable and are represented as

$$
\lambda^{(1)}(\varepsilon)=\lambda_{0}+\varepsilon \lambda_{1}^{(1)}+O\left(\varepsilon^{3 / 2}\right), \quad \lambda^{(2)}(\varepsilon)=\lambda_{0}+\varepsilon \lambda_{1}^{(2)}+O\left(\varepsilon^{3 / 2}\right) .
$$

In the considered case there exist two pairs of linearly independent vectors $e, g \in \mathbb{C}^{N}$ and $e^{*}, g^{*} \in \mathbb{C}^{N}$ such that

$$
A_{0} e=\lambda_{0} e, \quad A_{0} g=\lambda_{0} g, \quad A_{0}^{*} e^{*}=\overline{\lambda_{0}} e^{*}, \quad A_{0}^{*} g^{*}=\overline{\lambda_{0}} g^{*} .
$$

The vectors $e, g, e^{*}, g^{*}$ can be normalized in accordance with the identities

$$
\left(e, e^{*}\right)=\left(g, g^{*}\right)=1, \quad\left(e, g^{*}\right)=\left(g, e^{*}\right)=0 .
$$

Theorem 7.1. The coefficients $\lambda_{1}^{(1)}$ and $\lambda_{1}^{(2)}$ in formulae (7.1) are the eigenvalues of the matrix

$$
D=\left[\begin{array}{ll}
\left(A_{1} e, e^{*}\right) & \left(A_{1} g, e^{*}\right) \\
\left(A_{1} e, g^{*}\right) & \left(A_{1} g, g^{*}\right)
\end{array}\right]
$$

where $A_{1}=A^{\prime}(0)$.

Now suppose that the matrix $A_{0}=A(0)$ possesses a non-semi-simple double eigenvalue $\lambda_{0}$ (real or complex). Then for small $|\varepsilon|$ the matrix $A(\varepsilon)$ has two eigenvalues $\lambda^{(1)}(\varepsilon)$ and $\lambda^{(2)}(\varepsilon)$ such that $\lambda^{(1)}(0)=\lambda^{(2)}(0)=\lambda_{0}$. These functions are continuous and can be represented by Puiseux expansions:

$$
\lambda^{(1)}(\varepsilon)=\lambda_{0}+\varepsilon^{1 / 2} \lambda_{1}^{(1)}+O(\varepsilon), \quad \lambda^{(2)}(\varepsilon)=\lambda_{0}+\varepsilon^{1 / 2} \lambda_{1}^{(2)}+O(\varepsilon) .
$$

In the considered case, there exist two pairs of linearly independent vectors $e, g \in \mathbb{C}^{N}$ and $e^{*}, g^{*} \in \mathbb{C}^{N}$ such that

$$
A_{0} e=\lambda_{0} e, \quad A_{0} g=\lambda_{0} g+e, \quad A_{0}^{*} e^{*}=\overline{\lambda_{0}} e^{*}, \quad A_{0}^{*} g^{*}=\overline{\lambda_{0}} g^{*}+e^{*} .
$$

The vectors $e, g, e^{*}, g^{*}$ can be normalized according to the identities:

$$
\left(e, g^{*}\right)=\left(g, e^{*}\right)=1, \quad\left(e, e^{*}\right)=\left(g, g^{*}\right)=0 .
$$

Theorem 7.2. The coefficients $\lambda_{1}^{(1)}$ and $\lambda_{1}^{(2)}$ in expansions (7.4) are the numbers

$$
\lambda_{1}^{(1)}=\sqrt{\left(A_{1} e, e^{*}\right)}, \quad \lambda_{1}^{(2)}=-\lambda_{1}^{(1)} ;
$$

here $A_{1}=A^{\prime}(0)$.

Without explicit refereeing, we shall make use of the following properties of matrix $(1.2)$ :

$$
\operatorname{det} J=\operatorname{det} J^{-1}=1, \quad J^{-1}=J^{*}=-J, \quad \operatorname{Re}(J x, x)=0 \quad \text { for all } \quad x \in \mathbb{C}^{2 N} .
$$


7.2. Proof of Theorems 3.1 and 3.2. We restrict ourselves by proving Theorem 3.1; Theorem 3.2 can be proved in the same way.

By Theorem 7.1, the coefficients $\mu_{1}^{(1)}$ and $\mu_{1}^{(2)}$ in expansions 3.6 are the eigenvalues of the matrix

$$
D=\left[\begin{array}{ll}
\left(V_{1} e, e^{*}\right) & \left(V_{1} g, e^{*}\right) \\
\left(V_{1} e, g^{*}\right) & \left(V_{1} g, g^{*}\right)
\end{array}\right]
$$

here $V_{1}=V^{\prime}(0)$.

For the sake of definiteness, we suppose that under the assumptions of Theorem 3.1 normalization (3.2) holds. Then as the vectors $e^{*}$ and $g^{*}$ in matrix (7.6) we employ

$$
e^{*}=-i J e, \quad g^{*}=-i J g,
$$

where $e$ and $g$ are the vectors from (3.1). Vectors $(7.7)$ are linearly independent eigenvectors of the matrix $\left(J A_{0}\right)^{*}$ associated with the eigenvalue $\lambda=-\omega_{0} i$, that is, they satisfy the identities

$$
\left(J A_{0}\right)^{*} e^{*}=-\omega_{0} i e^{*}, \quad\left(J A_{0}\right)^{*} g^{*}=-\omega_{0} i g^{*} .
$$

By identities (3.2) and (3.5), the vectors $e, g, e^{*}, g^{*}$ satisfy normalization conditions (7.2).

The monodromy matrix $V(\varepsilon)$ of system 2.5 can be represented as follows, see, for instance, [21]):

here $V_{0}=e^{J A_{0} T}$

$$
V(\varepsilon)=V_{0}+\varepsilon V_{1}+V_{2}(\varepsilon)
$$

$$
V_{1}=V^{\prime}(0)=e^{J A_{0} T} \int_{0}^{T} e^{-J A_{0} \tau} J S_{1}(\tau) e^{J A_{0} \tau} d \tau,
$$

and $V_{2}(\varepsilon)$ is a continuously differentiable matrix satisfying the condition: $\left\|V_{2}(\varepsilon)\right\|=O\left(|\varepsilon|^{2}\right)$ as $\varepsilon \rightarrow 0$.

We substitute matrix (7.10) into (7.6) and taking into consideration identities (7.7), we complete the proof of Theorem 3.1 .

7.3. Proof of Theorem 3.3. By the vectors $e$ and $g$ in identities 3.16 we define new vectors:

$$
e_{1}=e, \quad g_{1}=\frac{1}{T \mu_{0}} g, \quad e_{1}^{*}=-\alpha J e, \quad g_{1}^{*}=\frac{1}{T \overline{\mu_{0}}} \alpha J g,
$$

where $\alpha$ is a non-zero coefficient, which is, in general, complex-valued. These vectors satisfy the identities:

$$
V_{0} e_{1}=\mu_{0} e_{1}, \quad V_{0} g_{1}=\mu_{0} g_{1}+e_{1}, \quad V_{0}^{*} e_{1}^{*}=\overline{\mu_{0}} e_{1}^{*}, \quad V_{0}^{*} g_{1}^{*}=\overline{\mu_{0}} g_{1}^{*}+e_{1}^{*} .
$$

At the same time, the vectors $e_{1}, g_{1}, e_{1}^{*}, g_{1}^{*}$ can be normalized in accordance with analogues of identities 7.5 by letting $\alpha=T \overline{\mu_{0}} /(e, J g)$.

To complete the proof of Theorem 3.3 it remains to apply Theorem 7.2 to matrix 7.9 .

7.4. Proof of Lemma 5.1. By vectors $e, g \in \mathbb{R}^{2 N}$ involved in identity (5.1) we define real vectors $e^{*}=J g$ and $g^{*}=J e$. Then

$$
\left(J A_{0}\right)^{*}\left(g^{*}+i e^{*}\right)=-\omega_{0} i\left(g^{*}+i e^{*}\right)
$$

that is, the vector $g^{*}+i e^{*}$ is an eigenvectors of the matrix $\left(J A_{0}\right)^{*}$ associated with the eigenvalue $\lambda=-\omega_{0} i$.

According to the spectral theory of linear operators, see, for instance, [19], the space $\mathbb{R}^{2 N}$ can be represented as a direct sumR $\mathbb{R}^{2 N}=E_{0} \oplus E^{0}$ of invariant subspaces of the operator $J A_{0}$. Here $E_{0}$ is the root subspace associated with the eigenvalues $\lambda_{0}= \pm \omega_{0} i$ of the operator $J A_{0}$, while $E^{0}$ is the root subspaces associated with the rest of the spectrum of this operator. The 
space $E_{0}$ is two-dimensional with a basis formed by $e$ and $g$. This space can be defined by the identity $E^{0}=\left\{v:\left(v, e^{*}\right)=\left(v, g^{*}\right)=0\right\}$.

Let us show that $\left|\left(e, e^{*}\right)\right|+\left|\left(e, g^{*}\right)\right|>0$. Indeed, assuming the opposite we get $\left(e, e^{*}\right)=$ $\left(e, g^{*}\right)=0$, that is, $e \in E^{0}$. On the other hand, by construction $e \in E_{0}$. This is possible only if $e=0$, but this contradicts to the fact that $e$ is an eigenvector.

To complete the proof of the lemma, it remains to note that $\left(e, e^{*}\right)=(e, J g)$ and $\left(e, g^{*}\right)=$ $(e, J e)=0$.

7.5. Proof of Theorem 5.1. By Theorem 7.1 the coefficients $\mu_{1}^{(1)}$ and $\mu_{1}^{(2)}$ in expansions (3.6) are the eigenvalues of matrix (7.6), in which $V_{1}=V^{\prime}(0)$ and the vectors $e$ and $g$ come from identity (5.1). As the vectors $e^{*}$ and $g^{*}$ we use the vectors

$$
e^{*}=\nu J g, \quad g^{*}=\nu J e,
$$

where $\nu$ is number $(5.2)$.

The entries of matrix (7.6) can be found by means of formulae (7.10) and (7.11). After appropriate transformations we obtain that matrix (7.6) coincides with matrix (5.3).

\section{BIBLIOGRAPHY}

1. A.P. Markeev. Linear Hamiltonian systems and some problems on stability of motion of satellite around the mass center. Inst. Komput. Issl., Moscow (2009). (in Russian).

2. V.A. Yakubovich, V.M. Starzhinskij. Linear differential equations with periodic coefficients. Nauka, Moscow (1972). [ John Wiley \& Sons, New York; Halsted Press Book, Jerusalem (1975).]

3. V.F. Zhuravlev, F.G. Petrov, M.M. Shunderyuk. Selected problems in Hamiltonian mechanics. Lenand, Moscow (2015). (in Russian).

4. V. Lanchares. On the stability of Hamiltonian dinamical systems // Monografias Matematicas Garca de Galdeano. 39, 155-166 (2014).

5. K. Meyer, G. Hall, D. Offin. Introduction to Hamiltonian dynamical systems and the $n$-body problem. Springer, New York (2009).

6. J. Moser. Lectures on Hamiltonian mechanics. Nauka, Moscow (1973). (in Russian).

7. V.A. Yakubovich, V.M. Starzhinski. Parametric resonance in linear systems. Nauka, Moscow (1987). (in Russian).

8. B.P. Demidovich. Lectures on mathematical stability theory. Nauka, Moscow (1967). (in Russian).

9. D. Treschev. Introduction to the perturbation theory of Hamiltonian systems. Fazis, Moscow (1998). (in Russian).

10. A.P. Seyranian, A.A. Mailybaev. Multiparameter stability theory with mechanical applications. World Scientific, New Jersey (2003).

11. A.P. Markeev. Libration points in celestial mechanics and cosmodynamics. Nauka, Moscow (1978). (in Russian).

12. A.P. Markeev. On a multiple resonance in linear Hamiltonian systems // Dokl. RAN. 402:3, 539-543 (2005). [Doklady Phys. 50:5, 278-282 (2005).]

13. A.D. Bruno. On types of stability in Hamilton systems // Preprint of Keldysh Inst. Appl. Math. 021, 24 pp. (2020). (in Russian).

14. A.D. Bruno. Normal form of Hamilton system with periodic perturbation // Preprint of Keldysh Inst. Appl. Math. 057, 27 pp. (2019). (in Russian).

15. O.V. Kholostova. On periodic motions of a nonautonomous Hamiltonian system in one case of multiple parametric resonance // Nelinein. Dinam. 13:4, 477-504 (2017). (in Russian).

16. B.S. Bardin, E.A. Chekina. On the constructive algorithm for stability investigation of an equilibrium point of a periodic Hamiltonian system with two degrees of freedom in first-order resonance case // Prikl. Mat. Mekh. 82:4, 414-426 (2018). [Mech. Solids. 53. Suppl. 2. 15-25 (2018).]

17. A. Elipe, V. Lanchares, A. Pascual. On the stability of equilibria in two-degrees of freedom hamiltonian systems under resonances // J. Nonl. Sci. 19, 305-319 (2005). 
18. L. Cesari. Asymptotic behavior and stability problems in ordinary differential equations. SpringerVerlag, Berlin (1963).

19. T. Kato. Perturbation theory for linear operators. Springer-Verlag, Berlin (1966).

20. L.S. Ibragimova L.S. I.G. Fanina, M.G. Yumagulov. The asymptotic formulae for the problem of the construction hyperbolic and stability regions of dynamical systems // Ufimskij Matem. Zhurn. 8:3, 59-81 (2016). [Ufa Math. J. 8:3, 58-78 (2016).]

21. M.G. Yumagulov, L.S. Ibragimova, A.S. Belova. Methods for studying the stability of linear periodic systems depending on a small parameter // Itogi Nauk. Tekhn. Ser. Sovrem. Mat. Pril. Temat. Obz. 163, 113-126 (2019). (in Russian).

22. A.B. Batkhin, A.D. Bruno, V.P. Varin. Stability sets of multi-parametric Hamiltonian systems // Preprints of Keldysh Inst. Appl. Math. 42, 32 pp. (2011). (in Russian).

23. G.E. Roberts. Linear stability of the elliptic lagrangian triangle solutions in the three-body problem // J. Diff. Eqs. 182:1, 191-218 (2002).

24. T. Kovacs. Stability chart of the triangular points in the elliptic restricted problem of three bodies // Mon. Not. R. Astron. Soc. 430:4, 2755-2760 (2013).

25. M.G. Yumagulov, O.N. Belikova, and N.R. Isanbaeva. Bifurcation near boundaries of regions of stability of libration points in the three-body problem // Astron. Zhurn. 95:2, 158-168 (2018). [Astron. Rep. 62:2, 144-153 (2018).

Marat Gayazovich Yumagulov,

Bashkir State University,

Zaki Validi str. 32,

450074, Ufa, Russia

E-mail: yum_mg@mail.ru

Lilia Sunagatovna Ibragimova,

Bashkir State University,

Zaki Validi str. 32,

450074, Ufa, Russia

E-mail: lilibr@mail.ru

Anna Sergeevna Belova,

Bashkir State University,

Zaki Validi str. 32,

450074, Ufa, Russia

E-mail: 89177662488@mail.ru 